\title{
Philosophiques
}

\section{En découvrant la métaphysique avec Jean Grondin}

\section{Peter Odabachian}

Volume 41, numéro 2, automne 2014

URI : https://id.erudit.org/iderudit/1027225ar

DOI : https://doi.org/10.7202/1027225ar

Aller au sommaire du numéro

Éditeur(s)

Société de philosophie du Québec

ISSN

0316-2923 (imprimé)

1492-1391 (numérique)

Découvrir la revue

Citer ce document

Odabachian, P. (2014). En découvrant la métaphysique avec Jean Grondin.

Philosophiques, 41(2), 365-369. https://doi.org/10.7202/1027225ar d'utilisation que vous pouvez consulter en ligne.

https://apropos.erudit.org/fr/usagers/politique-dutilisation/ 


\title{
En découvrant la métaphysique avec Jean Grondin
}

\author{
PETER ODABACHIAN \\ Département de philosophie \\ Collège Édouard-Montpetit
}

La non-assimilation du vécu, voire parfois l'impossibilité de l'assimiler constitue, je crois, le vécu caractéristique et incomparable du $\mathrm{Xx}^{\mathrm{e}}$ siècle ${ }^{1}$.

Que la métaphysique soit «un entretien de longue durée sur le sens des choses $^{2}$ ", Jean Grondin l'avait déjà lumineusement montré dans son Introduction à la métaphysique - au titre bien heideggérien, on l'aura remarqué -, l'ouvrage de 2004 qu'il dédiait à tous ses étudiants. Dix ans plus tard, j'oserais dire que si, comme l'écrit son auteur, «les idées en métaphysique vieillissent peu », lui-même me paraît avoir gagné en vigueur, dans la mesure où son plaidoyer pour cette discipline et cette "aspiration " insiste peut-être encore plus fermement, bien que toujours avec souplesse et nuance, sur ce qu'il nous est permis d'espérer de notre découverte la plus originaire du sens (des choses).

J'aimerais y aller de quelques brèves remarques sur son plus récent ouvrage avec une pensée pour un enseignement que j'ai eu le privilège de connaître. Du sens des choses est composé de leçons, et l'on peut souvent y entendre le rythme entraînant de l'enseignement oral de M. Grondin. J'aimerais ici attirer l'attention sur trois dimensions de notre existence qui à la fois fondent l'idée de la métaphysique en même temps qu'elles permettent de serrer de près certains des moments de l'argumentation de l'auteur.

\section{L'éducation et la mort}

La métaphysique est un besoin naturel de la pensée humaine, martèle sans détour M. Grondin, renouant ainsi avec certaines des affirmations des géants de la métaphysique qu'il présente. Métaphysique A s'ouvre hardiment sur le désir naturel qu'ont les hommes de savoir (connaître, comprendre). Kant parle de la philosophie comme d'une « disposition naturelle». On trouve sous la plume du tout jeune Hegel, confectionnant ses premières leçons à Iéna, l'expression de «besoin de l'universel». Et il nous arrive aussi d'oublier combien les propositions de la métaphysique sont audacieuses, voire iconoclastes. Combien l'interprétation et l'exposition de ce besoin ou de cette disposition n'a rien d'évident pour le sens commun, qui a tôt fait de déclarer que la philosophie, c'est le monde à l'envers.

1. I. Kertész, "Ce malheureux $\mathrm{xx}^{\mathrm{e}}$ siècle" (I995), dans L'Holocauste comme culture, Arles, Actes Sud, 2009.

2. Du sens des choses. L'Idée de la métaphysique, PUF, 20I3, I05. J'insérerai dorénavant les numéros de page entre parenthèses dans le corps du texte. 
Mais pourquoi se gênerait-on aujourd'hui ? Est-il si incongru de déclarer qu'on s'efforce de comprendre les racines et les causes des choses, ou qu'on puisse parler de notre "plus haute espérance» (44)? Non, avance Grondin — et j'applaudis cette franchise —, la métaphysique est nécessaire si l'on veut "pratiquer une philosophie qui sait ce qu'elle fait » (7). Et l'intelligence, lorsqu'il s'agit des choses essentielles, de la vérité, insistera-t-il aussi bellement en partant cette fois d'Homère, d'Héraclite et de Parménide, s'accompagne d'un «vif sentiment» (I I 2), d'une «intense émotion» (I I 6). Au cœur de l'ouvrage, dans son éclaircissement de la notion de vérité, l'auteur rappellera la dimension "d'effort, de conquête et de surpassement de soi » (99) que comporte l'atteinte d'une connaissance vraie (subtilement défendue comme «adéquate», dans ce contexte).

La métaphysique exige une patience qui jure avec l'empressement qui soutient le plus clair du temps nos projets de bonheur. Elle commande "pondération » et "méditation » (I 25). Les métaphysiciens que Grondin convoque, qu'ils en soient défenseurs ou détracteurs, le savent tous.

Car ce qui inquiète notre cœur est la recherche de meilleures raisons (3 I). Et si, "avant d'être des êtres de connaissance et d'action, nous sommes des êtres de pressentiment » (67), nous avons cependant à accomplir, à réaliser nos possibles. En ce sens, une connaissance philosophique peut à peu près toujours être révisée. "Malgré tous les revers qu'il peut essuyer, et qui n'ont pas à l'abattre, l'homme n'en fait pas moins l'expérience d'un sens qui précède certainement son intelligence et peut ressusciter son émerveillement (I39).»

L'auteur annonce d'emblée qu'il se servira de plusieurs illustrations et exemples médicaux (VIII). Avant Descartes, pour qui la médecine (de medeor: soigner) est l'une des branches de l'arbre de la philosophie, enracinée dans la métaphysique, ces illustrations souvent bien choisies nous rapprochent d'Aristote et des Grecs tout court, pour lesquels la philosophie reste toujours une thérapeutique, un soin de l'âme et du monde. En même temps, ils montrent combien notre expérience première des choses, à commencer par celle de notre corps, n'en est pas une d'indifférence, mais d'engagement et de déchiffrement inquiet, voire angoissé. S'il peut s'agir d'abord du corps, première passerelle vers la lumière, il en va plus remarquablement encore de ce qui ne sont devenus parfois pour nous que des mots. En effet, ce que peuvent vouloir dire le bien, le vrai, le beau ou le juste (classiquement: des "transcendantaux"), que nous nous y attardions de manière plus ou moins expresse, plus ou moins réfléchie, nous n'y sommes jamais indifférents. L'usage le plus commun de la langue en est traversé, il vaut la peine de s'en aviser et de se demander pourquoi, ici au sens directionnel, c'est-à-dire ce vers quoi le beau, par exemple, nous oriente.

Et la mort nous rappelle que l'être tout court ne nous est pas indifférent. J'ai beau pouvoir discuter légèrement (de l'être; des choses), voire bavarder, me plonger dans la possibilité de ma propre mort (mon propre 
non-être) peut me rappeler ma vraie et réelle tension au monde. On sait que c'est là un des effets dramatiques de la lecture d'Être et temps et de la position centrale qu'y occupe l'être-pour-la-mort. On se souvient aussi que la tremblante découverte de la mort, "le maître absolu ", constitue une étape cruciale dans la genèse de la conscience de soi dans la Phénoménologie de l'esprit, de Hegel. Et en creusant encore dans la tradition de nos textes de métaphysique, on retrouve évidemment la passionnante discussion de Socrate dans le Phédon, commentée par M. Grondin (40). S'il est pertinent et juste d'y mettre en relief la discussion de l'hypothèse de l'idée $(98 e)$, n'estil pas aussi impératif de souligner que Socrate, prisonnier dans sa cellule, est lui-même en train de transiger avec la mort? Lorsque Socrate se dit insatisfait de la lecture qu'on lui a faite d'Anaxagore, alors qu'il avait espéré en apprendre plus sur les causes intelligentes, ordonnatrices et universelles, n'est-il pas essentiel, avant tout, de dire qu'il a décidé d'affronter la mort et qu'il est dès lors insuffisant de dire qu'il ne se trouve là, en ce lieu, la cellule, que parce qu'il serait composé de "matière ", c'est-à-dire de chair, de muscles et d'os! Si l'amour de la sagesse, de même que tout engagement réel de l'homme, ajouterais-je, est, comme l'écrit l'auteur, un "effort» (2I), voire un « risque » (23), en voilà un exemple fondateur! Et si la métaphysique est une discipline, c'est-à-dire si elle peut s'apprendre (discere), elle est peut-être d'abord et avant tout une éducation à la mort, apprentissage qui nous fait souvent cruellement défaut.

Certains des dialogues de Platon, on le sait, y insistent plus théâtralement que les classifications parfois sèches qu'opère Aristote dans les textes dont nous avons hérité. Pensons en tout premier lieu au Banquet, où, si la soudaine apparition du beau est une promesse d'immortalité, l'expérience de la beauté reste toujours liée à notre fugacité.

Il tombe sous le sens que l'on peut à cet égard autant nommer les philosophes qu'invoquer toutes les sagesses du patrimoine culturel de l'humanité pour s'y éveiller. À ce titre, il me semble que M. Grondin ne fait parfois que peu de cas de cette mortalité, de notre finitude, sauf lorsqu'il est ques-

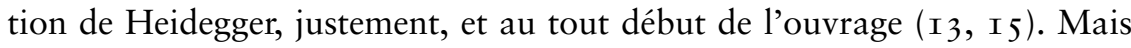
n'est-ce pas la mort, la Grande Chose (Rilke) qui reste pourtant l'inéluctable et fondatrice énigme de notre humanité, et à partir de laquelle toute connaissance dépassant le sensible peut être pensée, espérée ? Peut-être, je suppose, n'y aura-t-on pas insisté ici afin de laisser parler un peu plus la transcendance qui peut nous être impartie, pour peu qu'on s'y montre sensible.

\section{Apparence, illusion, égarement}

Du sens des choses s'adresse, à mon modeste avis, parfois davantage aux philosophes oublieux des sources natives de leur discipline qu'aux débutants en philosophie - que nous demeurons tous, sait bien l'auteur, enveloppés que nous sommes par le réel. Mais les étudiants en philosophie sont en principe ouverts et disposés à la radicalité de la protè philosophia, et, par 
intuition autant que par essence - notre humanité même - enclins à se laisser entraîner dans la découverte du plus fondamental. M. Grondin paraît lui-même le reconnaître lorsqu'il déplore dans sa sixième leçon que nombre de livres de "métamétaphysique » négligent de traiter de ces questions (I 25), c'est-à-dire de celles du sens.

Cela passe certes par l'apprentissage du lexique de la métaphysique, entendue maintenant comme discours. C'est un vocabulaire qui, tout en cherchant à dévoiler, a parfois aussi tôt fait d'obscurcir et de recouvrir les choses, si bien qu'alors - je reprends ici à mon compte une belle expression de l'auteur - la connaissance "trépigne au sein d'elle-même» (93).

On ne lui en fera certes pas le reproche, mais l'on doit observer que M. Grondin avance dans son argumentation avec la confiance dans le logos qu'on admire chez Socrate autant qu'on peut la retrouver, plus près de nous, chez un Gadamer. Mais peut-être faut-il souligner que nous avons plusieurs raisons de nous méfier du langage et de son (més)usage (aujourd'hui). J'y reviendrai bientôt. Cela ne constituera certes pas, j'en conviens, un argument contre la philosophie ou la métaphysique.

Discutons cependant de cette tromperie, et de l'illusion constitutive de notre expérience. Notre langage lui-même est truffé d'illusions, tissé d'apparences. Grondin le rappelle lorsqu'il discute du destinal Poème de Parménide et de la rencontre du jeune héros avec la déesse (I I 4 sq.). Il faut se méfier des mots et des noms. Et nos certitudes sensibles se renversent souvent d'elles-mêmes en leur contraire! Ainsi en peut-il aller avec des faux amis: une entente, même de longue date, peut avoir reposé sur un énorme malentendu.

Il en va de même lorsqu'il s'agit de la beauté, et qu'elle se découvre dans la «nature ", ou dans les choses fabriquées par l'homme - il se pourrait que cette différence entre les choses «naturelles » et les choses fabriquées par «nous » n'ait pas été suffisamment marquée, à mon sens, dans l'ouvrage. Mais l'expérience de la beauté est par essence un chemin d'erreur et d'égarement. C'est le fait de s'être laissé duper qui constitue la plupart du temps l'expérience, pour autant, j'en conviendrai, qu'on en tente le déchiffrement.

Il n'est pas sûr que nous sachions toujours ce que nous indiquent la musique, le théâtre ou la poésie. La possibilité que nous n’y entendions rien de rien reste toujours ouverte. Il se peut aussi que nous ne voulions rien savoir de ce qu'ils rendent présent. On se demandera pourquoi, d'un même tenant, nous avons tant besoin de voir représenter la laideur et la méchanceté. Qu'est-ce que cela indique sur ce que nous sommes?

Enfin, l'argumentation de l'auteur se veut souvent anamnétique, et non seulement dans la section qui porte ce nom: "Anamnèse, les fonds de la vérité » (73). Grondin est fidèle à la vocation anamnétique de la philosophie, ou de la métaphysique, lorsqu'il écrit que "la philosophie est affaire de réminiscence» (56). En témoigne Platon, mais cette pratique ou courant de 
fond va jusqu'à Hegel, ou même Heidegger, comme l'a déjà écrit l'auteur ${ }^{3}$. Les deux derniers, parmi les métaphysiciens les plus sensibles à l'histoire de la philosophie et à notre historicité tout court, ont déployé des pensées qui incluent l'erreur ou l'errance, voire la négativité ou le négatif, dans l'expérience (philosophique). Plutôt, c'est le négatif lui-même qui fonde l'expérience.

Laisser ouverte la possibilité de l'errance absolue, assumer cet égarement, ne peut-il pas être porteur de sens?

\section{Du sens des choses aujourd'hui}

Grondin cherche à rappeler le sujet du langage «à une époque, écrit-il, qui en a perdu le sens» (68). En effet, l'une des présuppositions de l'argumentation du Sens des choses, qui n'est pas toujours explicite mais quelquefois ouvertement affirmée, est celle selon laquelle «la philosophie, sinon le monde lui-même, a[urait] largement perdu le sens des choses " (63).

D'une part, il n'y a pas que la philosophie, comprise ici comme entreprise instituée, qui se soit quelquefois entortillée dans ses déconstructions internes, si bien qu'elle en oublierait sa tâche ou s'assujettirait parfois complaisamment à des exigences qui lui sont hétéronomes, qu'elles soient sociales, du marché, ou quoi encore. Mais il est vrai aussi, d'autre part, que les propositions de sens ne manquent pas (aujourd'hui). Les forces qui font concurrence à l'amour de la sagesse sont légion, et peut-être plus que jamais.

Je crois qu'il vaut la peine d'élucider davantage les raisons pour lesquelles nous aurions perdu ce sens de nous-mêmes, des choses et du monde. Quelles seraient donc les causes de notre difficulté à nous identifier à notre propre monde et à nous y reconnaître? Je suis d'accord avec l'idée qu'il faille pourfendre les insuffisances du dogme du constructivisme tous azimuts, ou encore une intelligence purement nominaliste du langage qui s'est répandue dans notre modernité et a colonisé notre compréhension du langage. Pourquoi est-ce, enfin, une compréhension (certes, très souvent idéologique) que semble préférer notre époque?

Enfin, ce qu'exige en tout cas Jean Grondin est la probité et la responsabilité d'assumer ses propositions et d'en légitimer la validité, ce qu'il fait à mon sens avec courage. Car si nous avons à être responsables, je le concèderai toujours, c'est notre monde partagé qui nous le rappelle avant tout.

3. Voir J. Grondin, «Pourquoi réveiller la question de l'être? », dans Heidegger. L'énigme de l'être, J.-F. Mattéi (dir.), Paris, PUF, 2004, 43-68, où M. Grondin écrit dès l'ouverture que "toute grande pensée depuis Parménide et Platon est anamnétique». 